\title{
Modelling Intravasation of Liquid Distension Media in Surgical Simulators
}

\author{
S. Tuchschmid ${ }^{1}$, M. Bajka ${ }^{2}$, D. Szczerba ${ }^{1}$, B. Lloyd ${ }^{1}$, G. Székely ${ }^{1}$, \\ and M.Harders ${ }^{1}$ \\ ${ }^{1}$ Computer Vision Laboratory, ETH Zurich, Switzerland \\ \{tuchschmid, domi, blloyd, szekely, mharders\}@vision.ee.ethz.ch \\ ${ }^{2}$ Clinic of Gynecology, University Hospital Zurich, Switzerland \\ michael.bajka@hin.ch
}

\begin{abstract}
We simulate the intravasation of liquid distention media into the systemic circulation as it occurs during hysteroscopy and transurethral resection of the prostate. A linear network flow model is extended with a correction for non-newtonian blood behaviour in small vessels and an appropriate handling of vessel compliance. We then integrate a fast lookup scheme in order to allow for real-time simulation. Cutting of tissue is accounted for by adjusting pressure boundary conditions for all cut vessels. We investigate the influence of changing distention fluid pressure settings and of the position of tissue cuts. Our simulation predicts significant intravasation only on the venous side, and just in cases when larger veins are cut. The implemented methods allow the realistic control of bleeding for short-term and the total resulting intravasation volume for long-term complication scenarios. While the simulation is fast enough to support real-time training, it is also adequate for explaining intravasation effects which were previously observed on a phenomenological level only.
\end{abstract}

\section{Introduction}

In both transurethral resection of the prostate and operative hysteroscopy, excess absorption of the distention fluid is one of the most serious complications [12]. During the intervention, the organ cavity is filled with a non-electrolytic distention fluid in order to allow for monopolar electrosurgery and to improve overall visibility. Fluid overload occurs when too much distention fluid is pressed through openly cut vessels into the patient's body, a process called intravasation. The amount of intravasation is influenced by the intrauterine pressure, the number and size of vascular openings and the duration of the procedure. Patients suffering from hyponatremia resulting from intravasation are at risk for pulmonary edema, cerebral edema, and cardiovascular collapse. Therefore, intrauterine pressure must be controlled to maintain a balance between too much pressure, increasing intravasation and too little pressure, decreasing visibility. In order to provide a means for learning the associated skills, we have extended our recently developed surgical simulator for hysteroscopy with a model of the intravasation process. 


\section{Methods}

In order to model the intravasation process, we calculate the blood flow in an interconnected vascular system in dependence of changing boundary conditions. A fully interconnected network flow model was first used by Fatt [3] for petrochemical research of oil and water flow through reservoir rock. Since then, the model has also often been employed to estimate pressure and flow distribution in blood vessel networks (see [4.5] for an overview).

The vascularisation of the virtual surgical scene [6] is generated according to 7] relying on a postulated oxygen consumption of the tissue. This scheme was extended to ensure that the length and number of vessels are following Horton's law [8] which originates from the observation of river branching and states that the number and length of vessels of a particular order follows a geometric sequence. Although Horton's formulae only represent an approximation, they have been proven valid in various morphometric studies [5]. In our current growth framework, arterial and venous sections are grown as separate trees. To ensure that the flow at the arterial inlet $\left(Q_{\text {inlet }}\right)$ is equal to the flow at the venous outlet $\left(Q_{\text {outlet }}\right)$, all diameters of the latter system are adjusted with a factor equal to $\sqrt[4]{Q_{\text {inlet }} / Q_{\text {outlet }}}$.

Linear Intravasation Model. In the linear model, the network is simulated as a number of vessel segments which are formed as straight pipes connecting vessel nodes [5]. For all bifurcation nodes, conservation of mass requires that $\sum_{i=1}^{k} Q_{i j}=0$, with $Q_{i j}$ being the flow between vessel nodes $i$ and $j$, and $k$ the number of segments merging at node $j$. For laminar, steady flow in stiff, straight and uniform tubes, the flow can be calculated according to Poiseuille's law and depends on the vessel conductance $G_{i j}$

$$
Q_{i j}=\left(P_{i}-P_{j}\right) G_{i j}, \quad \text { with } G_{i j}=\frac{\pi}{128} \cdot \frac{D_{i j}^{4}}{\mu_{i j} L_{i j}}
$$

where $D_{i j}, L_{i j}$ and $\mu_{i j}$ are the diameter, length and viscosity between nodes $i$ and $j$.

While the viscosity is approximately constant for larger vessels, the FahraeusLindquist effect causes non-Newtonian behaviour for vessels with small diameters often found in microcirculation. Therefore, we use in this case the modified blood viscosity relationship proposed in [9], which depends on tube diameter and hematocrit. For a hematocrit of 0.45 , the apparent relative viscosity in dependence of the tube diameter $D[\mu \mathrm{m}]$ is given by

$$
\mu=220 \cdot e^{-1.3 D}+3.2-2.44 \cdot e^{-0.06 D^{0.645}}
$$

The set of node equations can be reduced to a set of linear equations for the nodal pressures once the conductances have been evaluated for the given vessel geometry. Pressure boundary conditions have to be set for the arterial inlet node, the venous outlet node, and all arteriole/venule end nodes. In matrix form the set of equations is

$$
\mathbf{G} \cdot \mathbf{P}=\mathbf{G}_{B} \mathbf{P}_{B}
$$


with $\mathbf{G}$ the matrix of conductances, $\mathbf{P}$ the column vector of the unknown pressures, and $\mathbf{G}_{B} \mathbf{P}_{B}$ the column vector of the conductances times the boundary pressures of their attached vessel segments. This sparse system of equations is solved by a specialized solver [10. When cutting through tissue, we simply remove the vessel segments that are cut and set the pressure boundary conditions of the neighboring nodes equal to the pressure of the distention fluid. We then solve (3) with the changed boundary conditions. The updated $Q_{\text {inlet }}$ and $Q_{\text {outlet }}$ indicate the value for intravasation into the arterial and venous system.

(a)

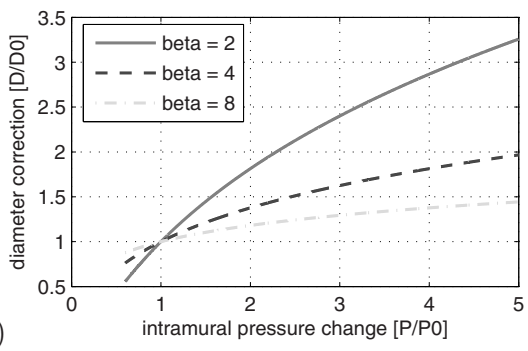

(b)

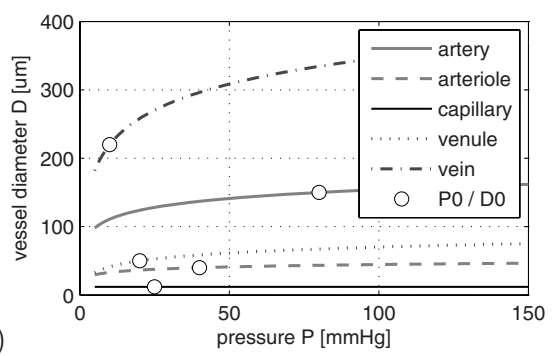

Fig. 1. Vessel diameter correction in dependence of changing pressure conditions for different stiffness indices (a) and different vessel types (b)

Compliant Intravasation Model. When vessels are cut, they are exposed to the differing pressure of the distention fluid, which causes a change in vessel diameter. Because of thinner walls and less connective tissue, the compliance of veins is considerably higher than for arteries [11. Therefore, the integration of a compliance model is especially important for the intravasation into the venous tree. We use the phenomenological description discussed in [12] for the pressurediameter relationship

$$
\ln \left(\frac{P}{P_{0}}\right)=\beta_{a / v}\left(\frac{D}{D_{0}}-1\right)
$$

where $P$ and $D$ are the updated pressure and diameter, and $P_{0}$ and $D_{0}$ are reference values and correspond to the pressure and diameter of the given vessel at a given operation point. The parameters $\beta_{a}$ and $\beta_{v}$ are the stiffness indices for arterial and venous vessels and are fitted to experimental data (e.g. [13]). In contrast to a linear compliance model, the stiffness index provides a better approximation for a larger range of pressures. Figure 1](a) shows the resulting diameter correction for different stiffness indices in dependence of the relative intramural pressure change. Figure 1(b) shows examples for vessels with various operating points $\left(P_{0} / D_{0}\right)$, with $\beta_{a}=8$ for arterial and $\beta_{v}=4$ for venous vessels. The dependence of the vessel conductance $G$ on the diameter $D$ (and therefore $P$ ) changes (3) to a non-linear system for the unknown pressures

$$
\mathbf{G}(\mathbf{P}) \cdot \mathbf{P}=\mathbf{G}(\mathbf{P})_{B} \mathbf{P}_{B}
$$

which we solve numerically by seeding the pressure variables with the linear solution, continue updating the diameters and conductances and iteratively solving 
the linear system until we reach steady-state conditions. Note that in the uncut model all vessel segments are at their respective operating points $\left(P=P_{0} / D=D_{0}\right)$, diameter corrections are all equal to one and the compliant model therefore identical to the linear model.

Real-time Simulation. The full non-linear flow system consists of 19'144 vessel segments with diameters ranging from $220 \mu \mathrm{m}$ for the venous outlet to $16 \mu \mathrm{m}$ for the capillaries. Solving the full reference system for flow and pressure takes around 2 seconds on a dual-core $3 \mathrm{GHz}$ PC. To speed up computation, we first calculate pressure values for all vessel nodes in the reference system. We then select a threshold for the minimal simulated vessel diameter. Next, we crop all smaller vessel segments and set the pressure boundary condition of the new end nodes to the precomputed values. Therefore, we only have to solve the reduced vessel system during run-time simulation. This scheme allows a balance between level of detail and computation time when real-time capability is more important than accuracy.

\section{Results}

Figure 4 depicts the vessel system used in all experiments. In Fig. 4(a), the initial pressure distribution is shown. Figure 4(b) shows the resulting pressure distribution after a horizontal cut. The nomenclature and default values for all used variables are shown in Table 1 . Reference values for all pressure boundary conditions are taken from literature data [1].

Table 1. Used variables and default values

\begin{tabular}{c|c|c} 
& description & default value \\
\hline$P_{\text {inlet/outlet }}$ & pressure at arterial inlet/venous outlet & $80 / 10 \mathrm{mmHg}$ \\
$D_{\text {inlet/outlet }}$ & diameter at arterial inlet/venous outlet & $150 / 221 \mu \mathrm{m}$ \\
$P_{\text {art/ven }}$ & pressure at arteriole/venule & $40 / 20 \mathrm{mmHg}$ \\
$D_{\text {art/ven }}$ & diameter of arteriole/venule & $16 \mu \mathrm{m}$ \\
$P_{\text {distention }}$ & pressure of distention fluid & $100 \mathrm{mmHg}$ \\
$\beta_{a / v}$ & stiffness index arteries/veins & $8.0 / 4.0$
\end{tabular}

The maximum pressure of the distention media $P_{\text {distention }}$ is set by the surgeon. While the recommended pressure range is between 60 and $100 \mathrm{mmHg}$ [14, we have experienced cases where necessary settings for clear view were up to $150 \mathrm{mmHg}$. The influence of changing distention fluid pressure from 10 to 150 $\mathrm{mmHg}$ for the cut shown in Fig. 4(b) is depicted in Fig. 2 For the approximation (3), the intravasation is a linear function of $P_{\text {distention }}$ as expected. $Q_{\text {inlet }}$ is negative for small pressure values, thus indicating bleeding. While the differences between the compliant and the linear model are small for $Q_{\text {inlet }}$ due to the high $\beta$-value for arteries, the intravasation into the venous system $Q_{\text {outlet }}$ is up to four times higher for the compliant model. For $P_{\text {distention }}=100 \mathrm{mmHg}$, the amount of 
(a)
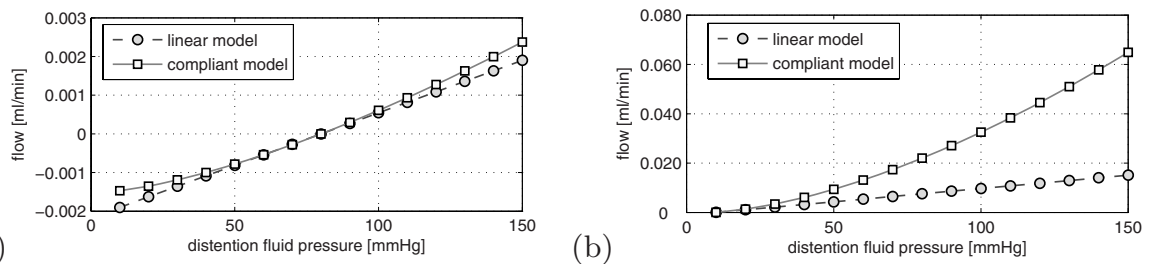

Fig. 2. Intravasation depending on distention fluid pressure for arterial inlet (a) and venous outlet (b)

(a)

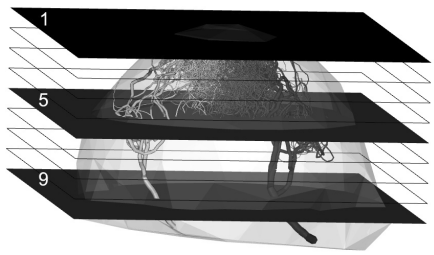

(b)

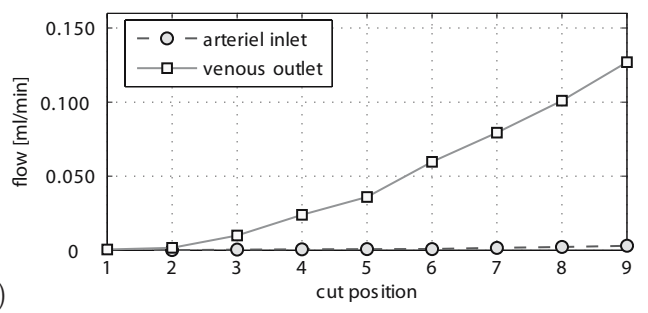

Fig. 3. Intravasation with compliant model (b) depending on cut position indicated by figure (a)

(a)
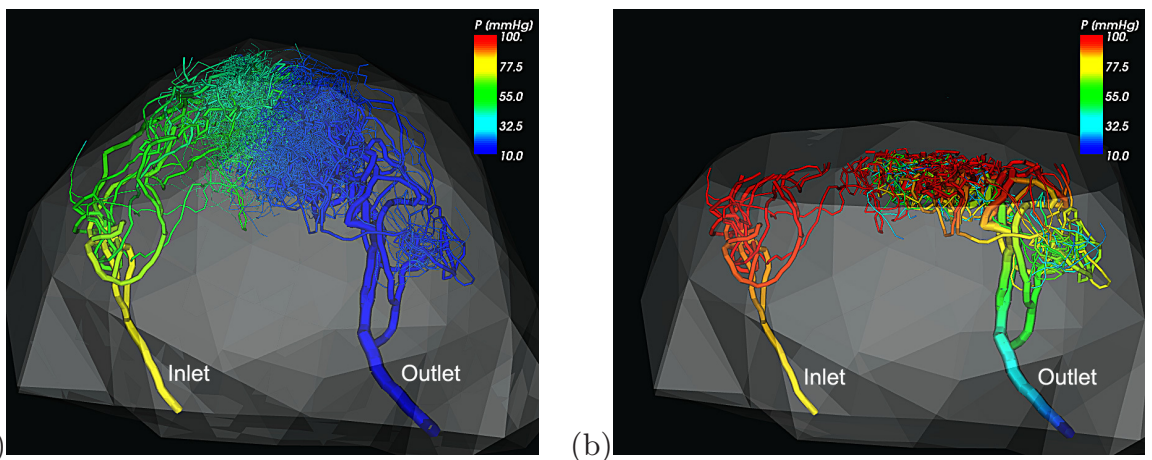

Fig. 4. Pressure distribution in myoma vascularisation before (a) and after (b) cut

intravasation into the venous system $\left(Q_{\text {outlet }}\right)$ is about 50 times larger than the flow into the arterial system $\left(Q_{\text {inlet }}\right)$.

According to medical literature, the amount of intravasation depends mainly on the size of cut vessels [14. By changing the position of the horizontal cut, we investigated the influence of superficial vs. deep cuts on $Q_{\text {outlet }}$ and $Q_{\text {inlet }}$. The cut positions are depicted in Fig. 3(a), while the resulting intravasation is displayed in Fig. 3(b). It can be clearly seen that significant intravasation only occurs into the venous system and only if larger vessels are cut.

We also investigated the necessary level of detail for the real-time simulation. With a minimal diameter for individually simulated vessel of $35 \mu \mathrm{m}$ and setting 
(a)

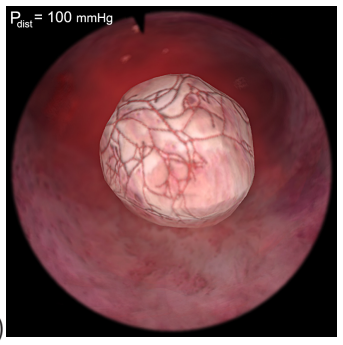

(d)

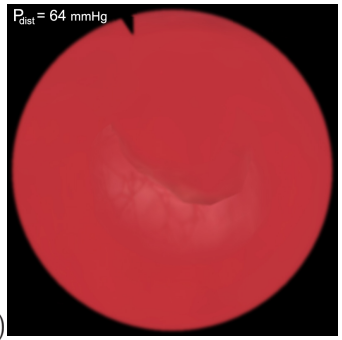

(b)

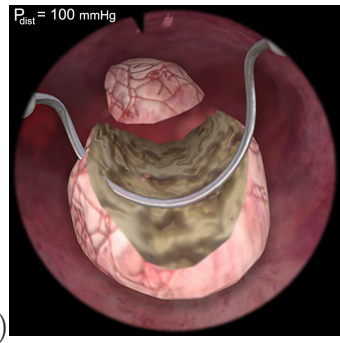

(e)

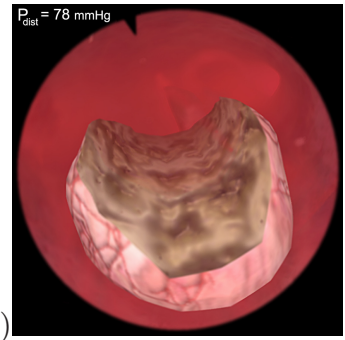

(c)

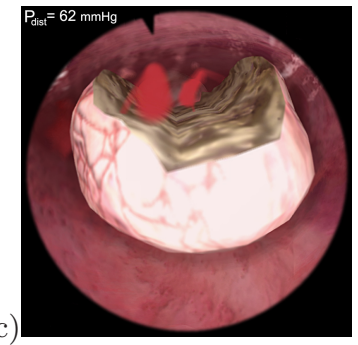

(f)

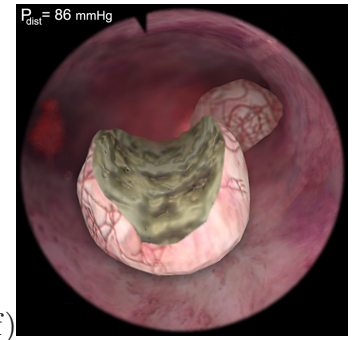

Fig. 5. Intravasation and bleeding simulation in the hysteroscopy simulator. The original situs with a myoma (a) has been cut (b) leading to bleeding (c) aggravated by the reduced distention fluid pressure ( $P_{\text {distention }}$ top left corner). The completely obscured view $(\mathrm{d})$ is then cleared by rinsing (e) leading to good visibility of the operation site (f).

the pressure boundary conditions to the initially computed values, the number of vessel segments was reduced from 19144 to 3839. This results in a computation speedup of 4.9 with a resulting error in $Q_{\text {outlet }}$ of just $1.6 \%$. Since we only have to update the simulation after a cut or when the distention fluid pressure has changed, the calculation time of 0.36 seconds is fast enough for real-time simulation in the training simulator. Figure 5 shows screenshots from an example intervention.

\section{Discussion}

A few of the assumptions of and possible extensions to our proposed model will be discussed in the following. Since the pressure of the distention fluid is in the same order of magnitude as the mean arterial pressure, the integration of a pulsatile flow model might be beneficial. However, we have not experienced any pulsating bleeding after cutting into a myoma. We also calculated Womersley and Reynolds numbers for all vessel segments and found them low enough to support the laminar flow model. In order to model flow behavior which departs from the parabolic velocity profile mandated by Poiseuille's law, a full three-dimensional finite element model would be necessary. However, the size of the full system with over 19'000 vessels prohibits the numerical solution with commercially available fluid solvers. While it would be possible to extend our model with correction factors based on a three-dimensional models for junctions and bended vessels, 
the literature shows that the expected effects are small for laminar flow and large length-diameter ratios [15].

Another open point is the effect of cutting into the vessel network. Due to the nature of electro-surgery, a thin layer of tissue is coagulated after a cut, effectively closing small vessels. In addition, small structures might buckle under the sudden pressure load. Also, vessels may deform after cutting because of residual wall stress. Since the discussed effects influence mainly the smallest vessel structures, their contribution to the overall intravasation might be even smaller than predicted by our model. The absolute value of the intravasation is highly dependent on the radii and overall morphometry of the vessel system (see Equation (1). A first visual comparison with data from a corrosion cast under electron microscopy [16] showed good agreement, yet no statistical analysis has been carried out. Unfortunately, there are no specific data available for the compliance in the vascular system of a myoma. However, while the absolute values for the resulting intravasation depend on diameter and compliance, the relative effects in changing cut position and distention fluid pressure are not affected.

\section{Conclusion and Future Work}

The presented framework enables the static analysis of pressure and flow distribution in large vessel networks. Based on the resulting flow, we control the bleeding for short-term complication scenarios in real-time. The intravasation volume and the resulting sodium serum level based on the performed surgery provide information on the long-term morbidity of the performed virtual surgery. According to our simulations, significant intravasation only occurs into the venous side, and only if larger veins are cut. While the simulation is fast enough to support real-time training, it is also adequate for explaining intravasation effects which were previously observed on a phenomenological level only.

Validation with real data is not meaningful without precise morphometric vessel data of the uterine cavitity, since we could simply tune the base vessel's diameter until we get a good agreement. With the availability of such data, we will extend our model to cover intravasation through the endometrium into the lymphatic system by diffusion as well as fluid uptake through the tubal ostia. Together this will then allow the quantitative comparison of the total intravasation volume with data from real surgery. Screenshots, movies of sample interventions and detailed descriptions of all simulator modules can be found on our project web (http://www.hystsim.ethz.ch).

Acknowledgment. This work has been performed within the NCCR Co-Me supported by the Swiss National Science Foundation.

\section{References}

1. Rassweiler, J., Teber, D., Kuntz, R., Hofmann, R.: Complications of transurethral resection of the prostate (TURP)-incidence, management, and prevention. Eur. Urol. 50(5), 969-979 (2006) 
2. Pasini, A., Belloni, C.: Intraoperative complications of 697 consecutive operative hysteroscopies. Minerva. Ginecol. 53(1), 13-20 (2001)

3. Fatt, I.: The network model of porous media: The dynamic properties of networks with tube radius distribution. Trans. AIME Petrol. Div. 207, 164-177 (1956)

4. Mayer, S.: On the pressure and flow-rate distributions in tree-like and arterialvenous networks. Bull. Math. Biol. 58(4), 753-785 (1996)

5. Kassab, G.S.: The coronary vasculature and its reconstruction. Ann. Biomed. Eng. 28(8), 903-915 (2000)

6. Sierra, R., Zsemlye, G., Székely, G., Bajka, M.: Generation of variable anatomical models for surgical training simulators. Med. Image. Anal. 10(2), 275-285 (2006)

7. Szczerba, D., Székely, G.: Simulating vascular systems in arbitrary anatomies. In: Duncan, J.S., Gerig, G. (eds.) MICCAI 2005. LNCS, vol. 3750, pp. 641-648. Springer, Heidelberg (2005)

8. Horton, R.: Erosional development of streams and their drainage basins: hydrophysical approach to quantitative morphology. Bull. Geol. Soc. Amer. 56, 275370 (1945)

9. Pries, A.R., Neuhaus, D., Gaehtgens, P.: Blood viscosity in tube flow: dependence on diameter and hematocrit. Am. J. Physiol. 263(6 Pt 2), H1770-H1778 (1992)

10. Davis, T.A.: Algorithm 832: Umfpack v4.3 - an unsymmetric-pattern multifrontal method. ACM Trans. Math. Softw. 30(2), 196-199 (2004)

11. Rooke, T.W., Sparks, H.V.: The Systemic Circulation, Medical Physiology, 2nd edn. ISBN: 0-7817-1936-4. Lippincott Williams \& Wilkins (2003)

12. Hayashi, K., Handa, H., Nagasawa, S., Okumura, A., Moritake, K.: Stiffness and elastic behavior of human intracranial and extracranial arteries. J. Biomech. 13(2), 175-184 (1980)

13. Kassab, G.S., Molloi, S.: Cross-sectional area and volume compliance of porcine left coronary arteries. Am. J. Physiol. Heart. Circ. Physiol. 281(2), H623-H628 (2001)

14. Menacaglia, L., Hamou, J.: Manual of Gynecological Hysteroscopy. Endo-Press, Tuttlingen (2001)

15. Perry, R., Green, D. (eds.): Perry's chemical engineers' handbook, 7th edn. McGraw-Hill, New York (1997)

16. Walocha, J.A., Litwin, J.A., Miodonski, A.J.: Vascular system of intramural leiomyomata revealed by corrosion casting and scanning electron microscopy. Hum. Reprod. 18(5), 1088-1093 (2003) 